\title{
Noncommutative spacetime, stringy spacetime uncertainty principle, and density fluctuations
}

\author{
Robert Brandenberger* \\ TH Division, CERN, CH-1211 Geneva 23, Switzerland \\ and Department of Physics, Brown University, Providence, Rhode Island 02912 \\ Pei-Ming $\mathrm{Ho}^{\dagger}$ \\ Department of Physics, National Taiwan University, Taipei 106, Taiwan, Republic of China
}

(Received 21 April 2002; published 22 July 2002)

\begin{abstract}
We propose a variation of spacetime noncommutative field theory to realize the stringy spacetime uncertainty relation without breaking any of the global symmetries of the homogeneous isotropic universe. We study the spectrum of metric perturbations in this model for a wide class of accelerating background cosmologies. Spacetime noncommutativity leads to a coupling between the fluctuation modes and the background cosmology which is nonlocal in time. For each mode, there is a critical time at which the spacetime uncertainty relation is saturated. This is the time when the mode is generated. These effects lead to a spectrum of fluctuations whose spectral index is different from what is obtained for commutative spacetime in the infrared region, but is unchanged in the ultraviolet region. In the special case of an exponentially expanding background, we find a scale-invariant spectrum. but with a different magnitude than in the context of commutative spacetime if the Hubble constant is above the string scale.
\end{abstract}

DOI: 10.1103/PhysRevD.66.023517

PACS number(s): 98.80.Cq, 98.70.Vc

\section{INTRODUCTION}

As a candidate for the theory of everything, string theory should tell us everything about the universe. One of the most important questions is how a successful theory of cosmology can be derived from it. Cosmology is becoming the major testing ground for string theory, since it tests the physics at energies much higher than can be reached in any collider on earth. On the other hand, cosmology also appears to require input from string theory, since cosmological theories based on classical gravity and the standard model of particle physics are expected to break down at very high energies (see e.g. [1] for a discussion of some of the conceptual problems of inflationary cosmology). There is a growing symbiotic relationship between string theory and cosmology (see e.g. [2]).

In this paper we are concerned with what string theory can say about cosmology. Obviously, we are very far from being able to derive cosmology directly from string theory. Instead of proposing a new scenario starting from a very special string theory configuration, we would like to focus on a universal property of string theory, and study its implication for cosmology. The universal property which we wish to focus on is the stringy spacetime uncertainty relation (SSUR), which states that the uncertainties in the physical time and space coordinates $\Delta t_{p}=\Delta t$ and $\Delta x_{p}$ satisfy

$$
\Delta t_{p} \Delta x_{p} \geqslant l_{s}^{2},
$$

where $l_{s}$ is the string length scale. Yoneya suggested [3] that the SSUR is a universal property for strings as well as D-branes. This is in contrast with the other stringy uncertainty relation [4]

\footnotetext{
*Email address: rhb@het.brown.edu

†Email address: pmho@phys.ntu.edu.tw
}

$$
\Delta x_{p} \Delta p \geqslant 1+l_{s}^{2} \Delta p^{2},
$$

which implies a minimal length scale

$$
\Delta x_{p} \geqslant l_{s}
$$

but does not hold for D-branes. In an earlier paper [5], it was argued that the SSUR can be used to solve the flatness and the horizon problems in cosmology without the need to invoke cosmic inflation.

In this paper, we study the effect of the SSUR on metric perturbations in the early universe, which are the origin of the observed large-scale structure and of cosmic microwave background anisotropies. The reason one expects signatures of Planck (string) scale physics in the spectrum of density fluctuations on cosmological scales today is the following $[1,6]$ : since the fluctuations on large scales are small today, and since gravity is a purely attractive force, the fluctuations had to be extremely small in the early Universe, thus justifying a linearized analysis. According to such an analysis, the individual Fourier modes of the fluctuation field evolve independently. In the context of an expanding background cosmology, there was thus a time when the physical wavelengths of modes which are probed in current cosmological experiments was smaller than the Planck (or string) scale, and hence one cannot neglect the effects of Planck (string) scale physics. ${ }^{1}$

As a first step, we consider a simple model in which matter is dominated by a single scalar field. As is done in inflationary cosmology, we quantize the joint linear metric and matter fluctuations about a classical homogeneous and iso-

\footnotetext{
${ }^{1}$ In this context of inflationary cosmology, this non-robustness of the predictions of inflation to possible effects of Planck (string) scale physics is known as the trans-Planckian problem.
} 
tropic background cosmology. With these assumptions the cosmological perturbations are automatically Gaussian.

In order to carry out the calculation explicitly, we need a specific model to realize the SSUR. Motivated by the recent developments on noncommutative geometry in string theory, we generalize the field theory on $(1+1)$-dimensional noncommutative space, where the uncertainty relation is a direct result of the spacetime noncommutativity, to $3+1$ dimensions.

We identify two crucial effects which lead to a difference between the evolution of fluctuations in commutative and noncommutative spacetimes. The first is a coupling between the fluctuation mode and the background which is nonlocal in time, and the second is the appearance of a critical time for each mode at which the SSUR is saturated, and which is taken to be the time when the mode is generated. ${ }^{2}$

These two effects lead to a change in the spectral index of the fluctuations in the infrared region for all accelerating background cosmologies except for the exponentially expanding case. Instead of a red spectrum (as is obtained in commutative spacetime) we obtain a blue spectrum. In the case of an exponentially expanding background, a scaleinvariant spectrum results.

\section{PRELIMINARIES}

In string theory, the best known example of noncommutative field theory is the low energy effective theory of D-branes in the background of a $B$ field [8]. It has also been proposed that Ramond-Ramond (RR) background fields may lead to a low energy effective theory of gravity best described as Einstein gravity living on a certain noncommutative spacetime [9]. Motivated by recent developments of noncommutative geometry in string theory, models for cosmological fluctuations on noncommutative spacetime have been proposed by several groups $[10,11] .{ }^{3}$ However, in these proposals, some isometries of the Friedmann-RobertsonWalker (FRW) metric are broken by the noncommutativity. In this paper, we will not give a specific commutation relation for spacetime. Rather, we will imagine that the SSUR [3] is realized by a deformation of the commutative field theory in a way which preserves all of the global symmetries of the classical background. One can imagine that, although their background expectation values vanish and thus there is no well-defined spacetime noncommutativity, quantum fluctuations of the $B$ field (for D-branes) or certain RR fields (for spacetime) would still result in spacetime uncertainty [14]. Keeping this in mind, we will first consider field theories on noncommutative space, which may give us a hint about the result of "averaging over" (in a path integral) the effective action for different background fields.

The formulation of general relativity on noncommutative

\footnotetext{
${ }^{2}$ Note that this implies that the Hilbert space of the perturbative quantum theory of gravity becomes time-dependent. This appears to be a natural consequence of any attempt to quantize gravity in the context of cosmology, as discussed in [7] and in references therein.

${ }^{3}$ Models based on Eq. (2) are also studied [12,13].
}

spacetime has been extensively studied [15-17]. The notions of metric, distance, etc. can all be defined. It is therefore natural for us to assume that the notion of metric still makes sense as long as it is consistent with SSUR. Assuming a homogeneous isotropic background, we take the FriedmannRobertson-Walker (FRW) metric

$$
d s^{2}=d t^{2}-a^{2}(t)\left(\frac{d r^{2}}{1-K r^{2}}-r^{2} d \Omega^{2}\right) .
$$

For simplicity, we will focus on the case of a spatially flat universe $(K=0)$. For later use, we introduce another time coordinate $\tau$ (not the usual conformal time) such that the metric is

$$
d s^{2}=d t^{2}-a^{2}(t) d x^{2}=a^{-2}(\tau) d \tau^{2}-a^{2}(\tau) d x^{2} .
$$

Since the scale factor has no spatial dependence, the SSUR imposes no restriction on the Hubble constant $H \equiv \dot{a} / a$, or any other scale associated with the scale factor, such as $\dot{H}^{1 / 2}$. On the other hand, the constraint (3) implies that $H<M_{s}$, and $\dot{H}<M_{s}^{2}$, etc., where $M_{s}=l_{s}^{-1}$ is the string energy scale. As we will see below, this difference is crucial for us.

In view of the connection between noncommutative field theory and SSUR described above, the fact that SSUR does not impose a restriction on quantities with only time dependence can be understood via noncommutative field theory. Obviously, if we only look for solutions with a single variable, noncommutative field theories are equivalent to commutative field theories.

In the case of a classical spacetime, for a given equation of state of matter $p=w \rho$ with constant $w$, the solution of the (classical) Einstein equations for the scale factor is

$$
a(t)=a_{0} t^{n}=\alpha_{0} \tau^{n /(n+1)},
$$

where

$$
n=\frac{2}{3(1+w)}
$$

and $\alpha_{0}=\left((n+1)^{n} a_{0}\right)^{1 /(n+1)}$. For an expanding universe, $n$ $>0$. For a matter dominated universe, $w=0$ and $n=2 / 3$; for a radiation dominated universe, $w=1 / 3$ and $n=1 / 2$. The universe accelerates if $n>1$. If the cosmological constant dominates, then $w=-1, a(t)=a_{0} \exp (H t)(n=\infty)$ and we have exponential inflation $[18,19]$.

Since we do not have the exact gravitational theory with all the stringy corrections to Einstein gravity, we will consider a generic expanding scale factor $a$. As we review in the Appendix, for a given scale factor $a$, the metric perturbations for gravity coupled to a single scalar field obey an equation of motion of the form

$$
\mu_{k}^{\prime \prime}+\left(k^{2}-\frac{z_{k}^{\prime \prime}}{z_{k}}\right) \mu_{k}=0
$$

where the primes mean derivatives with respect to a certain time coordinate $\tilde{\eta}$ to be defined below. (In the commutative 
case, $\tilde{\eta}=\eta$ is the conformal time defined by $d t=a d \eta$.) This equation applies both to gravitational waves (in which case $\mu=a h, h$ being the amplitude of the gravitational wave, and - for commutative spacetime $-z_{k}=a$ ) and to scalar metric fluctuations, the fluctuations which couple to matter [in which case $a^{-1} \mu$ is the scalar field fluctuation in the uniform spatial curvature gauge and-in commutative spacetime$z_{k}=z$ is a function describing the background model and is given explicitly by Eq. (A9)]. For backgrounds in which the equation of state does not change in time (the ones considered here), the function $z$ for scalar metric fluctuations is proportional to $a$, and hence the equations for scalar metric fluctuations and gravitational waves coincide.

A quantity used frequently to compare theory with observations is the power spectrum, which measures the strength of the fluctuations on the scale $k$. The power spectrum of metric perturbations is given by

$$
P_{k}=\frac{k^{3}}{2 \pi^{2}} \frac{\left|\mu_{k}\right|^{2}}{z_{k}^{2}} .
$$

(To allow comparison with what is done for a noncommutative spacetime, we have allowed $z$ to depend on $k$ ) If the background is expanding, then as explained in the Appendix (with the normalization factor chosen to apply to gravitational waves),

$$
P(k) \simeq k^{2} z_{k}^{-2}\left(\tilde{\eta}_{k}\right) M_{P}^{-2}
$$

where $M_{P}$ denotes the Planck mass, and $\tilde{\eta}_{k}$ is the earliest time when

$$
\frac{z_{k}^{\prime \prime}}{z_{k}}>k^{2} .
$$

In the usual expanding cosmological models described by the ansatz (6), then for a commutative spacetime, we have $z_{k}=a$. Hence, for $n>1$, the time $\tilde{\eta}_{k}$ is the time when the scale $k$ exits the Hubble radius, and we have $\eta_{k} \propto k^{-1}$, and thus $z_{k}\left(\eta_{k}\right) \propto k^{n /(1-n)}$. Therefore, the spectrum of gravitational waves and of scalar metric fluctuations obeys

$$
P_{k} \simeq c k^{2 /(1-n)},
$$

where $c=\left(\left(2 n^{2}-n\right)^{n} a_{0}^{2}\right)^{1 /(n-1)}$. For the spectrum to be exactly scale invariant, we need $n=\infty$, that is, exponential inflation, for which the spectrum is

$$
P_{k} \simeq \frac{H^{2}}{M_{P}^{2}} .
$$

\section{TOY MODEL ON NONCOMMUTATIVE SPACETIME}

First we consider the case of $1+1$ dimensions where the SSUR can be easily realized by the * product, so that we can get some hint about how the SSUR affects the action of a scalar field.

The SSUR

$$
\Delta t \Delta x_{p}=\Delta \tau \Delta x \geqslant l_{s}^{2} .
$$

can be realized by the algebra of noncommutative spacetime

$$
[\tau, x]_{*}=i l_{s}^{2} .
$$

This * product can be explicitly defined as

$$
\left(f^{*} g\right)(x, \eta)=\left.e^{-(i / 2) l_{s}^{2}\left(\partial_{x} \partial_{\tau^{\prime}}-\partial_{\tau} \partial_{y}\right)} f(x, \tau) g\left(y, \tau^{\prime}\right)\right|_{y=x, \tau^{\prime}=\tau} .
$$

The fact that the algebra (15) is time independent makes $\tau$ a better coordinate than the conformal time $\eta$ defined by $d t$ $=a d \eta$, which is frequently used when calculating metric perturbations. Naively, the SSUR can be written as

$$
\Delta \eta \Delta x \geqslant \frac{l_{s}^{2}}{a^{2}(\eta)}
$$

in terms of $\eta$. However, this relation is not well defined when $\Delta \eta$ is large, because the argument $\eta$ for the scale factor on the right hand side changes over the time interval $\Delta \eta$, and it is thus not clear what to use for $a(\eta)$ in Eq. (17).

A comment on unitarity is in need at this point. Since spacetime noncommutativity introduces higher derivatives of time in the Lagrangian of a field theory, a naive treatment will often result in loss of unitarity. (But there are also examples [20] for which the unitarity is not broken by spacetime noncommutativity.) However, in our case, the fundamental theory is string theory, and we are looking at its effective field theory (with focus on the stringy effect described by the SSUR). It is very common for effective theories to have higher derivatives terms, and a proper treatment [21] should preserve unitarity order by order perturbatively. Furthermore, the field theory we will consider below is essentially a free field theory, and so we will not have to worry about unitarity in this paper.

As discussed in the Appendix, both scalar metric fluctuations and gravitational waves are described by free scalar field actions on the classical expanding background (in two spacetime dimensions there are obviously no gravitational waves). We will now propose a noncommutative field theory which generalizes the action for cosmological perturbations to the case of noncommutative space-time. We will start from the actions expressed in terms of the observable fields $h$ and $\mathcal{R}$ in the case of gravitational waves and scalar metric fluctuations, respectively (see the Appendix). In the case of scalar metric fluctuations the variable $\mathcal{R}$ is the fluctuation of the spatial curvature in the comoving gauge in which $\delta \varphi$ $=0$. As can be deduced from Eqs. (A2) and (A10), respectively, these are free field actions except that the expansion of the background cosmology has not been factored out (i.e. the equations of motion for these variables contain the Hubble damping terms). These actions thus contain a nontrivial measure factor $a^{d-1}$ or $z^{d-1}$, in the cases of gravitational waves and scalar metric fluctuations, respectively, where $d$ is the number of spatial dimensions. We will extract a factor of $a^{-2}$ from the measure and insert it into the operator appearing in the Lagrangian to account for the fact that 
the spatial gradient appearing in the operator should reduce to the usual operator in Minkowski space-time when expressed in terms of physical distances. We propose to make the transition to noncommutative spacetime obeying the SSUR by taking the operator appearing in the action and replacing all multiplications by $*$ products.

Based on the above considerations, we take the free field action for a real scalar field in $1+1$ dimensions

$$
\widetilde{S}=\int d \tau d x \frac{1}{2}\left(\partial_{\tau} \phi^{\dagger *} a^{2 *} \partial_{\tau} \phi-\left(\partial_{x} \phi\right)^{\dagger *} a^{-2 *}\left(\partial_{x} \phi\right)\right) .
$$

In terms of the Fourier transform of $\phi$,

$$
\phi(\tau, x)=V^{1 / 2} \int_{k<k_{0}(\tau)} \frac{d k}{2 \pi} \phi_{k}(\tau) e^{i k x}
$$

(where $V$ is the total spatial coordinate volume), the action is

$$
\widetilde{S} \simeq V \int_{k<k_{0}} d \tau d k \frac{1}{2}\left(\beta_{k}^{+} \partial_{\tau} \phi_{-k} \partial_{\tau} \phi_{k}-k^{2} \beta_{k}^{-} \phi_{-k} \phi_{k}\right),
$$

where

$$
\beta_{k}^{ \pm}(\tau)=\frac{1}{2}\left(a^{ \pm 2}\left(\tau-l_{s}^{2} k\right)+a^{ \pm 2}\left(\tau+l_{s}^{2} k\right)\right) .
$$

The * product in the action takes care of the SSUR for the interaction between the background metric and the scalar field. In order to realize the SSUR for the scalar field by itself, we have imposed an upper bound on the comoving momentum $k$ at $k_{0}$ in Eq. (19). The reason is as follows. In order for a fluctuation mode with wave number $k$ to exist, the SSUR must be satisfied. According to Eq. (20), the energy defined with respect to $\tau$ for a mode $k$ is

$$
E_{k}=k a_{e f f}^{-2},
$$

where

$$
a_{e f f}^{2}=\left(\frac{\beta_{k}^{+}}{\beta_{k}^{-}}\right)^{1 / 2} .
$$

Using $\Delta x \sim 1 / k, \Delta \tau \sim 1 / E_{k}$ together with the SSUR, we find

$$
\left(\frac{a_{e f f}(\tau)}{k}\right)^{2} \sim \Delta x_{p} \Delta t \geqslant l_{s}^{2}
$$

and thus we have an upper bound on the wave number

$$
k \leqslant k_{0}(\tau) \equiv \frac{a_{e f f}(\tau)}{l_{s}} .
$$

One should also check whether the background metric satisfies the SSUR by itself. Yet as we mentioned above, the SSUR imposes no contraint on the background since it is homogeneous.
Because of the SSUR, the mode $\phi_{k}$ interacts with the background with an uncertainty of $l_{s}^{2} k$ in $\tau$. In general, if the uncertainty relation is not realized exactly by the commutation relation (15), we expect that $\beta_{k}^{ \pm}$will be replaced by functions of the form

$$
\beta_{k}^{ \pm}(\tau)=\int d \xi f(\xi) a^{-2}(\tau-\xi k)
$$

for some even function $f(\xi)$ peaked at the origin with a characteristic width $l_{s}^{2}$. By Taylor expansion, we have

$$
\beta_{k}^{ \pm}(\tau)=\left(1+C_{1}^{ \pm} \frac{H p}{M_{s}^{2}}+C_{2}^{ \pm} \frac{\left(\dot{H}+H^{2}\right) p^{2}}{M_{s}^{4}}+\cdots\right) a^{ \pm 2}(\tau),
$$

where $C^{ \pm}$are constants of order 1 , and $p=k / a$ is the physical momentum. This is a special case of the most general correction due to new physics at an energy scale $M_{s}$, which can be expanded in powers of $\left(H / M_{s}\right)^{2}$ and $\left(p / M_{s}\right)^{2}$ independently [22].

The action (20) reduces to the action for metric fluctuations (A2) on classical spacetime when $l_{s} \rightarrow 0$.

To calculate the power spectrum, it is convenient to rewrite the action in the form

$$
\widetilde{S} \simeq V \int_{k<k_{0}} d \tilde{\eta} d k \frac{1}{2} y_{k}^{2}(\tilde{\eta})\left(\phi_{-k}^{\prime} \phi_{k}^{\prime}-k^{2} \phi_{-k} \phi_{k}\right),
$$

where the new time coordinate $\tilde{\eta}$ is defined by

$$
\frac{d \tilde{\eta}}{d \tau}=\left(\frac{\beta_{k}^{-}}{\beta_{k}^{+}}\right)^{1 / 2}=a_{e f f}^{-2}
$$

and

$$
y_{k}=\left(\beta_{k}^{-} \beta_{k}^{+}\right)^{1 / 4} \text {. }
$$

The primes mean derivatives with respect to $\tilde{\eta}$.

\section{THE MODEL}

The previous section motivates a model to incorporate the SSUR for any spacetime dimension:

$$
S=V \int_{k<k_{0}} d \tilde{\eta} d^{d} k \frac{1}{2} z_{k}^{d-1}(\tilde{\eta})\left(\phi_{-k}^{\prime} \phi_{k}^{\prime}-k^{2} \phi_{-k} \phi_{k}\right),
$$

where $z_{k}$ is some smeared version of $z$ or $a$ over a range of time of characteristic scale $\Delta \tau=l_{s}^{2} k$. As $\tau$ increases, the effect of the shift $\Delta \tau$ for a given mode becomes less important. The time coordiante $\tilde{\eta}$ is related to $\tau$ by $d \tilde{\eta}=\tilde{z}_{k}^{-2} d \tau$, where $\tilde{z}_{k}$ is another smeared version of $a$, As an example, suppose that the only difference between the $d+1$ dimensional action and the $(1+1)$-dimensional one (28) is the measure $z^{d-1}$ for the additional $(d-1)$ dimensions, then we have 


$$
z_{k}^{d-1}(\tilde{\eta})=z^{d-1} y_{k}^{2}(\tilde{\eta}), \quad \tilde{z}_{k}(\tilde{\eta})=a_{e f f}(\tilde{\eta})
$$

with $y_{k}$ given by Eq. (30), and $a_{e f f}$ by Eq. (23). In the case of gravitational waves, the function $z_{k}$ is denoted $a_{k}$, with $a_{k}$ constructed from the scale factor $a$ in the same way as $z_{k}$ is obtained from $z$, say, in Eq. (32).

This deformation has the advantage of preserving both spatial translational and rotational symmetry of the (flat) FRW metric, in constrast with constructions based on the commutation relations

$$
\left[x^{\mu}, x^{\nu}\right]=i \theta^{\mu \nu} .
$$

We can now turn to the calculation of the spectrum of cosmological fluctuations in various expanding background cosmologies, including inflationary backgrounds. We emphasize here that except the flat FRW metric, so far we have not assumed anything about the stringy correction to the Einstein gravity. A key role in which the SSUR enters in the analysis of fluctuations is the existence of a characteristic time $\tilde{\eta}_{k}^{0}$ for each mode $k$ which is the time when the SSUR is saturated

$$
k=k_{0}\left(\widetilde{\eta}_{k}^{0}\right)
$$

According to Eq. (25), the mode k cannot exist before $\tilde{\eta}_{k}^{0}$.

A major issue is in which state the fluctuations are generated. On scales which are smaller than the Hubble radius at the time of formation, the distinguished choice is the local vacuum state (the state which appears empty of particles in the comoving frame at the time of formation). But it is unclear when should be the time to impose this initial condition. For modes which are generated when the wavelength is greater than the Hubble length, the choice is usually even less clear. However, in our case, the choice is obvious for all modes. At $\tilde{\eta}<\tilde{\eta}_{k}^{0}$, the fluctuation mode $k$ does not exist. By continuity of $N_{k}$, the operator representing the number of quanta of the $k$ th mode measured with respect to the adiabatic vacuum (see e.g. [23] for a textbook discussion of these concepts), then when the mode first becomes physical at $\tilde{\eta}$ $=\tilde{\eta}_{k}^{0}$, it must be in the vacuum state. Our choice is thus to consider the amplitude of the growing mode at the time of formation to be the same as the function would have in the vacuum state in the absence of cosmological expansion.

As will be derived below, the fluctuations have a different spectral index in two wavelength regions which we call UV and IR, respectively. The UV modes are generated within the Hubble radius, and it turns out that for these modes the approximation (35) holds, The IR modes are generated outside the Hubble radius, where the effect of the SSUR is important, and the approximation (35) breaks down.

We first consider the $U V$ region, namely values of $k$ for which $\Delta \tau$ is small in the sense that all smeared versions of $a$ can be approximated by $a$. As in Eq. (27), the smeared scale factors $z_{k}, \tilde{z}_{k}$ are expansions of $\left(H p / M_{s}^{2}\right)^{2}$ (and $\dot{H} p^{2} / M_{s}^{4}$ ) etc. The approximation

$$
z_{k} \simeq \tilde{z}_{k} \simeq a
$$

is valid when

$$
H p \ll M_{s}^{2} .
$$

As $a$ increases with time, this approximation gets better and better.

For this bound to be true at all times for a mode $k$, we only need to make sure that it is satisfied when the mode first appeared at $\tilde{\eta}_{k}^{0}$. Since $\Delta x_{p}=\Delta t_{p} \simeq 1 / p$, the saturation of the SSUR at $\tilde{\eta}_{k}^{0}$ implies that

$$
p=M_{s}
$$

at $\widetilde{\eta}_{k}^{0}$. Hence, Eq. (36) is equivalent to

$$
H\left(\tilde{\eta}_{k}^{0}\right) \ll M_{s} .
$$

For the ansatz (6), as an example, the approximation (35) is good if

$$
k \gg A \equiv \alpha_{0}^{n+1} l_{s}^{n-1} .
$$

From Eq. (10), the perturbation spectrum is determined by the earliest time when Eq. (11) is satisfied. In the absence of the cutoff (25), this is the time when

$$
\frac{z_{k}^{\prime \prime}}{z_{k}} \simeq k^{2}
$$

Assuming Eq. (35), this condition can be rewritten as

$$
\dot{H}+2 H^{2} \simeq p^{2}
$$

For $n>1$ (the accelerating case), this occurs roughly when the size of the fluctuation crosses the Hubble radius, i.e.,

$$
H\left(\tilde{\eta}_{k}\right) \simeq p
$$

From Eq. (38), we see that $\tilde{\eta}_{k}^{0} \leqslant \tilde{\eta}_{k}$. This means that the fluctuations are generated inside of the Hubble radius, and this was the initial definition of the UV region. Thus, it is presicely in the UV region that the approximation (35) is valid.

Since the UR modes are generated on scales inside the Hubble radius in their local vacuum state, and since the evolution of the modes after that is no different than in the case of a commutative spacetime, it follows immediately that the spectrum for the UV modes (39) is the same as the classical case (12). The uncertainty relation has no significant effect for these modes, in agreement with the general consideration of [22].

Now we would like to study the $I R$ modes $k \ll A$ which are generated outside the Hubble radius, and for which the effects of the SSUR are important. Although our discussion about the IR modes will be more speculative because the Hubble expansion rate is above the string scale at the time of IR mode formation, the following provides an interesting example showing how new physics at the string scale can have significant effect on the spectrum of perturbations, 
evading the pessimistic conclusion of [22]. ${ }^{4}$ As we will see, our description of the SSUR results in a spectrum which except in the case of exponential inflation has a different spectral index than what would be obtained in commutative space-time.

As an example, we take $z_{k}$ and $\tilde{z}_{k}$ given by Eq. (32) for $z=a$. The first step in the analysis is to obtain the expression for $\tau_{k}^{0}$. Starting point is Eq. (25) which defines the initial time. Inserting the expression (23) for $a_{\text {eff }}^{2}$ and the formulas (21) for $\beta_{k}^{ \pm}$, and then using Eq. (6) for the scale factor, we obtain

$$
\tau_{k}^{0}=\left(\left(\frac{k l_{s}}{\alpha_{0}}\right)^{2(n+1) / n}+k^{2} l_{s}^{4}\right)^{1 / 2} .
$$

For the IR modes $k$ which we are focusing on, i.e. $k \ll A$, the second term dominates over the first term. Thus, we have $\beta_{k}^{+} \simeq \frac{1}{2} a^{2}\left(\tau+l_{s}^{2} k\right)$ and $\beta_{k}^{-} \simeq \frac{1}{2} a^{-2}\left(\tau-l_{s}^{2} k\right)$ (in the case of an expanding universe).

To find the power spectrum for these modes, we start from Eq. (10) with the time $\tilde{\eta}_{k}$ replaced by the time $\tilde{\eta}_{k}^{0}$ when the modes are generated. We then use Eq. (32) to replace $z_{k}^{2}$ by the product of $z^{2}$ and $y_{k}$, and then insert the expression for $y_{k}$ from Eq. (30). After inserting the above approximate expressions for $\beta_{k}^{ \pm}$we obtain

$$
P_{k} \sim k^{2} a^{-2}\left(\tau_{k}^{0}\right) a^{-1 / 2}\left(\tau_{k}^{0}+l_{s}^{2} k\right) a^{1 / 2}\left(\tau_{k}^{0}-l_{s}^{2} k\right) .
$$

It is apparent that the nonlocal coupling between background and fluctuation mode has a large effect. Making use of Eq. (43) [and keeping in mind that the second term in Eq. (43) dominates over the first one], the final result becomes

$$
P_{k}=c^{\prime} k^{3 /(n+1)},
$$

where

$$
c^{\prime}=\frac{M_{s}^{(5 n-1) /(n+1)}}{2^{(n-1) / 2(n+1)} \alpha_{0}^{3} M_{P}^{2}} .
$$

This is obviously very different from the old result (12). Instead of a red spectrum we now obtain a blue spectrum.

To summarize, for an accelerating universe $(n>1)$, the spectrum has a negative spectral index in the UV region, but a positive one in the IR region. A smooth interpolation between the two regions would yield a nearly flat spectrum in the transition region. Note that as $n$ increases, the slope of the power spectrum decreases. Thus, the more rapidly the Universe is accelerating, the closer the spectrum is to being scale-invariant. This is similar to what occurs in ordinary power law inflation, except that the sign of the spectral index is opposite. In the limit towards exponential inflation, the results for commutative and non-commutative space-times converge.

\footnotetext{
${ }^{4}$ After completion of this manuscript two papers appeared which reach similar conlusions concerning the potential observability of trans-Planckian physics in the spectrum of fluctuations [24,25].
}

The case of exponential inflation is very special in that we do not need to specify $z_{k}$ or $\tilde{z}_{k}$ to determine the index of the power spectrum. Despite the fact that $z_{k}(\tilde{\eta})$ and $\tilde{z}_{k}(\tilde{\eta})$ are different from $a$, their dependence on $k$ happens to be the same as $a\left(\tilde{\eta}_{k}^{0}\right): z_{k}\left(\tilde{\eta}_{k}^{0}\right) \propto \tilde{z}_{k}\left(\tilde{\eta}_{k}^{0}\right) \propto a\left(\tilde{\eta}_{k}^{0}\right) \propto k$. Therefore the spectrum is scale invariant, like in the commutative case. For $H \ll M_{s}$, the approximation (35) is valid, and the spectrum is roughly the same as Eq. (13). But for $H \gg M_{s}$, the magnitude can be different. For instance, if we take Eq. (32), it is

$$
P_{k} \simeq \frac{M_{s}^{5}}{\sqrt{2} H^{3} M_{P}^{2}} .
$$

If we assume that Eq. (13) is correct for the tensor metric perturbations, the observational bound for gravitational waves $P_{k}<10^{-10}$ leads to the usual hierarchy problem [26] $H<10^{-5} M_{P}$ of inflationary cosmology. But if Eq. (47) is correct, the problem is alleviated. For $H \simeq M_{P}$, we only need $M_{S}<10^{-2} M_{P}$.

\section{DISCUSSION}

We have studied the consequences of the SSUR on the evolution of cosmological fluctuations in expanding cosmological backgrounds. Given a noncommutative spacetime obeying the SSUR, the cosmological background will still be described by the Einstein equations since the background fields only depend on one spacetime variable. The equations for the linear fluctuations, however, are modified. We have argued that the modifications take the form of an interaction of the fluctuating field with the background which is nonlocal in time.

An important consequence of the SSUR is that for each comoving wave number $k$, there is an earliest time $\tilde{\eta}_{k}^{0}$ at which the fluctuating mode exists. We assume that the fluctuation starts out with its vacuum amplitude at this time. Since the dependence of $\eta_{k}^{0}$ on $k$ is nontrivial, we expect that the index of the power spectrum of cosmological fluctuations will be different than for commutative spacetime. We find that this is indeed the case for a range of wavelengths, except in the special case of exponential inflation in which we also obtain a scale-invariant spectrum. Note that for power law inflation $(n>1)$ the spectrum of fluctuations is "blue" when the SSUR is effective (for the infra red region $k<A$ ) in contrast to the case of a commutative spacetime in which the spectrum is "red." The reason for this difference is that in our case, modes with smaller values of $k$ are generated later than those with a larger value, and thus experience growth due to squeezing for less time, whereas for commutative spacetime the larger the value of $k$, the later the mode leaves the Hubble radius and the less squeezing it will experience.

Let us now conclude with a few remarks.

(1) Although we find the same requirement $m=-1$ as in the undeformed scalar field theory for a scale-invariant spectrum, it represents a special case because the spectrum is different for other values of $m$ in the IR.

(2) In the case of exponential inflation, although the spectrum is always roughly scale-invariant, the amplitudes are 
different for large $H$ and small $H$,

(3) It may appear a little counterintuitive that it is the IR fluctuation modes that are severly modified by the stringy spacetime uncertainty relation. This may be interpreted as a manifestation of the UV-IR connection.

(4) A realization of inflation in the context of noncommutative spacetime obeying the SSUR would not be subject to the trans-Planckian problem of general inflationary models [1]. The case of exponential inflation in our model can also be viewed as a test of the robustness of inflation, but with a different kind of deformation of the dispersion relation than those considered in [6].

(5) In the case of scalar metric fluctuations, we had to make a choice of which variable to consider as the one whose action is subject to the transformation from commutative spacetime to noncommutative spacetime outlined in Sec. III. It would be interesting to study the results for other choices.

(6) An intriguing possibility is that there is only one effective scale factor, i.e., $z_{k}=\tilde{z}_{k}$. With this assumption, we do not need the approximation (35) to calculate the spectrum. The spectrum (10) can be interpreted as the physical energy $E_{k}$ squared at $\tilde{\eta}_{k}^{0}$ for all modes with $\tilde{\eta}_{k}^{0}>\tilde{\eta}_{k}^{1}$. The SSUR implies that $E_{k}\left(\tilde{\eta}_{k}^{0}\right)=M_{s}$, and so we obtain

$$
P_{k} \simeq \frac{M_{s}^{2}}{M_{P}^{2}}
$$

for all UV modes (39).

\section{ACKNOWLEDGMENTS}

R.B. thanks Karim Benakli and Yaron Oz for useful discussions, and is grateful to Professor P. Hwang for the invitation to deliver a series of lectures in Taiwan. P.M.H. thanks Tzihong Chiueh, Je-An Gu, Hsien-chung Kao, Feng-Li Lin, Sanjaye Ramgoolam, and Hyun-Seok Yang for helpful discussions. The work of R.B. is supported in part by the U.S. Department of Energy under Contract DE-FG0291ER40688, TASK A, and by the CERN Theory Division. R.B. thanks the CERN Theory Division for hospitality during the course of this work. The work of P.M.H. is supported in part by the National Science Council, the CosPA project of the Ministry of Education, the National Center for Theoretical Sciences, Taiwan, R.O.C. and the Center for Theoretical Physics at National Taiwan University.

\section{APPENDIX: ESSENTIALS OF THE THEORY OF COSMOLOGICAL PERTURBATIONS}

For a detailed review of the calculation of metric perturbations, the reader is directed to other references [27,28] (see also [29] for a recent short review). Here we outline the basic steps with modifications due to noncommutative spacetime or SSUR effects.

There are two kinds of metric perturbations of interest in early Universe cosmology: the scalar and tensor fluctuations. Tensor fluctuations correspond to gravitational waves. The perturbed metric only has nonvanishing space-space components $h_{i j}$ which can be expanded in terms of the two basic traceless and symmetric polarization tensors $e_{i j}^{+}$and $e_{i j}^{x}$ as

$$
h_{i j}=h_{+} e_{i j}^{+}+h_{x} e_{i j}^{x}
$$

where the space and time dependence is in the coefficient functions $h_{+}$and $h_{x}$.

The Einstein action can be expanded to second order in the metric fluctuations about a Friedmann-Robertson-Walker (FRW) background (4), and the action for $h_{+}$and $h_{x}$ reduces to that of a free, massless, minimally coupled scalar field in the FRW background. To obtain the correct normalization, the metric must be multiplied by the normalization factor $M_{p l} / \sqrt{2}$. In Fourier space, the action is

$$
S=\int d \eta \frac{1}{2} a^{d-1}\left(\varphi_{-k}^{\prime} \varphi_{k}^{\prime}-k^{2} \varphi_{-k} \varphi_{k}\right),
$$

where $\varphi$ stands for the coefficient functions $h_{+}$and $h_{x}$, and $d$ denotes the number of spatial dimensions (set to 3 in the following in most of this Appendix). This leads to the equation of motion

$$
\varphi_{k}^{\prime \prime}+2 \frac{a^{\prime}}{a} \varphi_{k}^{\prime}+k^{2} \varphi_{k}=0
$$

The Hubble friction term can be eliminated via a change of variables

$$
\mu=a \varphi
$$

yielding the equation of motion

$$
\mu_{k}^{\prime \prime}+\left(k^{2}-\frac{a^{\prime \prime}}{a}\right) \mu_{k}=0 .
$$

The power spectrum of gravitational waves in a particular state $|0\rangle$ of the gravitational radiation field can be written in terms of the new field $\mu$ as

$$
\mathcal{P}_{g}(k)=2 \frac{k^{3}}{2 \pi^{2}} \frac{\left\langle 0\left|\mu_{k}^{*} \mu_{k}\right| 0\right\rangle}{a^{2}} .
$$

The two point function appearing in Eq. (A6) is that of a free canonically normalized massless scalar field multiplied by $2 / M_{p l}^{2}$.

Scalar metric fluctuations couple to matter, and give rise to the large-scale structure of the Universe. The description of scalar metric perturbations is more complicated than the analysis of gravitational waves both because of the coupling to matter and also because some perturbation modes correspond to space-time reparametrizations of a homogeneous and isotropic cosmology. This is the issue of gauge fixing. A simple way to address this issue is to work in a system of coordinates which completely fixes the gauge. A simple choice is the longitudinal gauge, in which the metric takes the form [27] 


$$
d s^{2}=a^{2}(\eta)\left[(1+2 \Phi) d \eta^{2}-(1-2 \Psi) \gamma^{i j} d x_{i} d x_{j}\right],
$$

where the space and time dependent functions $\Phi$ and $\Psi$ are the two physical metric degrees of freedom which describe scalar metric fluctuations ( $\gamma^{i j}$ is the metric of the unperturbed spatial hypersurfaces). The fluctuations of matter fields give additional degrees of freedom for scalar metric fluctuations. In the simple case of a single scalar matter field, the matter field fluctuation can be denoted by $\delta \varphi$. In the absence of anisotropic stress, it follows from the Einstein equations that the two metric fluctuation variables $\Phi$ and $\Psi$ coincide. Due to the Einstein constraint equation, the remaining metric fluctuation $\Psi$ is determined by the matter fluctuation $\delta \varphi$.

It is clear from this analysis of the physical degrees of freedom that the action for scalar metric fluctuations must be expressible in terms of the action of a single free scalar field $v$ with a time dependent mass (determined by the background cosmology). As shown in [30] (see also [31], this field is

$$
\begin{aligned}
v & =a\left(\delta \varphi+\frac{\varphi_{0}^{\prime}}{\mathcal{H}} \Psi\right) \\
& =z \mathcal{R},
\end{aligned}
$$

where $\varphi_{0}$ denotes the background value of the scalar matter field, $\mathcal{H}=a^{\prime} / a$,

$$
z=a \frac{\varphi_{0}^{\prime}}{\mathcal{H}}
$$

and $\mathcal{R}$ denotes the curvature perturbation in comoving gauge [32]. The action for scalar metric fluctuations is [33]

$$
S=\frac{1}{2} \int d^{4} x \sqrt{-\gamma}\left(v^{\prime 2}-\gamma^{i j} v_{, i} v_{, j}+\frac{z^{\prime \prime}}{z} v^{2}\right),
$$

(where $\gamma$ is the determinant of the metric $\gamma^{i j}$ ) which leads to the equation of motion

$$
v_{k}^{\prime \prime}+\left(k^{2}-\frac{z^{\prime \prime}}{z}\right) v_{k}=0
$$

which under the change $a \rightarrow z$ is identical to Eq. (A5) for gravitational waves. Note that if $a(\eta)$ is a power of $\eta$, then $\varphi_{0}^{\prime}$ and $\mathcal{H}$ scale with the same power of $\eta$ so that $z$ is proportional to $a$, in which case the evolution of gravitational waves and scalar metric fluctuations is identical.

In analogy to Eq. (A6), the power spectrum of the curvature fluctuation $\mathcal{R}$ is

$$
\mathcal{P}_{\mathcal{R}}(k)=\frac{k^{3}}{2 \pi^{2}} \frac{\left\langle 0\left|v_{k}^{*} v_{k}\right| 0\right\rangle}{z^{2}} .
$$

So far, the theory was developed for commuting space-time variables. To make the transition to non-commutative spacetime, and to take into account the space-time uncertainty relation, we replace, as discussed in the main part of the text, the $k$-independent function $z(\eta)$ by a $k$-dependent function $z_{k}(\eta)$. In addition, we allow the initial time $\eta^{0}$ to depend on $k$.

In order to show how the growth of the classical mode function $v_{k}$ translates into the growth of the expectation value which determines the power spectrum we will make use of the Hamiltonian formalism. From the action (A10) it follows that the momentum canonically conjugate to the field $v$ is

$$
\Pi_{k}=v_{-k}^{\prime}-\frac{z_{k}^{\prime}}{z_{k}} v_{-k},
$$

and this leads to the Hamiltonian (see e.g., [6])

$$
H=\int d^{d} k\left[k a_{k}^{\dagger} a_{k}+\frac{i}{2} \frac{z_{k}^{\prime}}{z_{k}}\left(a_{k}^{\dagger} a_{-k}^{\dagger}-a_{k} a_{-k}\right)\right],
$$

where $a_{k}^{\dagger}(\eta)$ and $a_{k}(\eta)$ are the creation and annihilation operators at time $\eta$ related to $v_{k}$ and its conjugate momentum by

$$
\begin{gathered}
v_{k}=\frac{1}{\sqrt{2 k}}\left(a_{k}+a_{-k}^{\dagger}\right), \\
\Pi_{k}=\frac{-i}{\sqrt{2 k}}\left(a_{-k}-a_{k}^{\dagger}\right) .
\end{gathered}
$$

In the Heisenberg picture, the creation and annihilation operators evolve with time, while the state does not. Assume that the Universe is in the state $|0\rangle$ defined by

$$
a_{k}\left(\eta_{k}^{0}\right)|0\rangle=0,
$$

which is the vacuum for mode $k$ at some intial time $\eta_{k}^{0}$. It is in general not the vacuum at later times.

The Bogoliubov transformation relates the creation and annihilation operators at $\eta^{0}$ with the corresponding operators at the time $\eta$ :

$$
\begin{aligned}
& a_{k}(\eta)=\alpha_{k}(\eta) a_{k}\left(\eta_{0}\right)+\beta_{k}(\eta) a_{-k}^{\dagger}\left(\eta_{0}\right), \\
& a_{k}^{\dagger}(\eta)=\bar{\beta}_{k}(\eta) a_{-k}\left(\eta_{0}\right)+\bar{\alpha}_{k}(\eta) a_{k}^{\dagger}\left(\eta_{0}\right),
\end{aligned}
$$

where $\alpha_{k}$ and $\beta_{k}$ satisfy

$$
\alpha_{k} \bar{\alpha}_{k}-\beta_{k} \bar{\beta}_{k}=1, \quad \forall k .
$$

The Hamilton equations $\left[H, a_{k}\right]=i \dot{a}_{k}$ and its Hermitian conjugate take on a simpler form when written in terms of the new variables

$$
\zeta_{k}=\alpha_{k}-\bar{\beta}_{k}, \quad \xi_{k}=\alpha_{k}+\bar{\beta}_{k} .
$$

In terms of them they read

$$
\zeta_{k}^{\prime \prime}+\left(k^{2}-\frac{z_{k}^{\prime \prime}}{z_{k}}\right) \zeta_{k}=0,
$$




$$
\xi_{k}^{\prime \prime}+\left(k^{2}-\frac{\left(z_{k}^{-1}\right)^{\prime \prime}}{\left(z_{k}^{-1}\right)}\right) \xi_{k}=0
$$

The larger the value of $\left|\beta_{k}\right|^{2}(\eta)$, the larger the number of particles at time $\eta$ created out of the initial vacuum state $|0\rangle$.

Let us denote the factors in Eqs. (A22), (A23) by

$$
M=k^{2}-\frac{z_{k}^{\prime \prime}}{z_{k}}, \quad N=k^{2}-\frac{\left(z_{k}^{-1}\right)^{\prime \prime}}{\left(z_{k}^{-1}\right)} .
$$

For small $\eta(\eta<-1 / k)$ (scales smaller than the Hubble radius), both $M$ and $N$ are positive and approximately equal to $k^{2}$. Thus, $\zeta_{k}$ and $\xi_{k}$ oscillate. If the initial state is taken to be the local vacuum state,

$$
\alpha_{k}\left(\eta_{0}\right)=1, \quad \beta_{k}\left(\eta_{0}\right)=0,
$$

then the magnitudes of $\zeta$ and $\xi$ are of order 1 until $\eta=$ $-1 / k$. This represents the oscillation of quantum vacuum fluctuations. We will refer to this regime as phase I. On scales larger than the Hubble radius (at later times), $M$ and $N$ are dominated by the second (negative) term. In this period, $\zeta$ and $\xi$ correspond to frozen fluctuations which are undergoing quantum squeezing and which scale like $z_{k} \sim a$ and $z_{k}^{-1} \sim a^{-1}$, respectively. Since $a$ is increasing with time, by Eq. (A21) we can approximate $v_{k}$ by $\zeta_{k} / \sqrt{2 k}$ for sufficiently late times. This is phase II. When $k^{2}$ is much smaller than both $z_{k}^{\prime \prime} / z_{k}$ and $\left(z_{k}^{-1}\right)^{\prime \prime} /\left(z_{k}^{-1}\right)$, and assuming that $z$ is an increasing function, the dominant solutions are simply

$$
\zeta_{k}=C_{k} z_{k}, \quad \xi_{k}=\frac{1}{C_{k} z_{k}}
$$

for real $u_{k}, v_{k}$. Note that the condition (A20) is satisfied for this solution, but not for other solutions of the second order differential equations. Suppose that for sufficiently late times, $z_{k} \gg 1\left(z_{k} \ll 1\right)$ then $v_{k} \simeq \frac{1}{2} \zeta_{k} / \sqrt{2 k} \quad\left(v_{k} \simeq \frac{1}{2} \xi_{k} / \sqrt{2 k}\right)$. Since the initial condition is $\zeta_{k}\left(\eta_{0}\right)=\xi_{k}\left(\eta_{0}\right)=1$, it is equivalent to say that for $z_{k} \gg 1$, for sufficiently large $\eta$, $v_{k}(\eta)$ is given by the solution to the differential equation (A22) with the initial condition $v\left(\eta_{0}\right)=1 / \sqrt{2 k}$. For $z_{k} \ll 1$, we replace $z_{k}$ by $z_{k}^{-1}$. In general we want to find the larger of the two functions $\zeta_{k}$ and $\xi_{k}$. This then determines via the relations(A21) the Bogoliubov coefficient $\beta_{k}$, in terms of which the power spectrum of metric fluctuations becomes

$$
\mathcal{P}_{\mathcal{R}}(k)=\frac{k^{3}}{2 \pi^{2}} \frac{1}{2 k} \frac{\left|\beta_{k}\right|^{2}}{z_{k}^{2}} .
$$

Note that the factor $1 /(2 k)$ represents the vacuum normalization of the states, seen in Eq. (A15).

In summary, for expanding $z_{k}$, the spectrum is

$$
\mathcal{P}_{\mathcal{R}}(k)=\frac{k^{2}}{4 \pi^{2}} \frac{1}{z_{k}^{2}\left(\eta_{k}\right)},
$$

where $\eta_{k}$ is the time when the fluctuation mode $k$ crosses the Hubble radius $(M=0)$. In this paper we also consider the case of fluctuations outside the Hubble radius starting in the vacuum. For them $\eta_{k}$ should be taken to be the time when they start in the vacuum.
[1] R.H. Brandenberger, "Inflationary cosmology: Progress and problems," hep-ph/9910410.

[2] R.H. Brandenberger, "The promise of string cosmology," hep-th/0103156.

[3] T. Yoneya, in Wandering in the Fields, edited by K. Kawarabayashi and A. Ukawa (World Scientific, Singapore, 1987), p. 419; M. Li and T. Yoneya, Phys. Rev. Lett. 78, 1219 (1997); T. Yoneya, Prog. Theor. Phys. 103, 1081 (2000).

[4] G. Veneziano, Europhys. Lett. 2, 199 (1986); D.J. Gross and P.F. Mende, Nucl. Phys. B303, 407 (1988); D. Amati, M. Ciafaloni, and G. Veneziano, Phys. Lett. B 216, 41 (1989); R. Guida, K. Konishi, and P. Provero, Mod. Phys. Lett. A 6, 1487 (1991).

[5] J.-A. Gu, P.-M. Ho, and S. Ramgoolam, "Stringy Spacetime Uncertainty as an Alternative to Inflation," hep-th/0101058.

[6] R.H. Brandenberger and J. Martin, Mod. Phys. Lett. A 16, 999 (2001); J. Martin and R.H. Brandenberger, Phys. Rev. D 63, 123501 (2001).

[7] T. Jacobson, Prog. Theor. Phys. Suppl. 136, 1 (1999).

[8] N. Seiberg and E. Witten, J. High Energy Phys. 09, 032 (1999).

[9] A. Jevicki and S. Ramgoolam, J. High Energy Phys. 04, 032
(1999); P.-M. Ho, S. Ramgoolam, and R. Tatar, Nucl. Phys. B573, 364 (2000); P.-M. Ho and M. Li, ibid. B596, 259 (2001); B590, 198 (2000).

[10] C.-S. Chu, B.R. Greene, and G. Shiu, Mod. Phys. Lett. A 16, 2231 (2001).

[11] S. Alexander, R. Brandenberger, and J. Magueijo, "Noncommutative inflation," hep-th/0108190.

[12] A. Kempf and J.C. Niemeyer, Phys. Rev. D 64, 103501 (2001).

[13] R. Easther, B.R. Greene, W.H. Kinney, and G. Shiu, Phys. Rev. D 64, 103502 (2001); "Imprints of short distance physics on inflationary cosmology," hep-th/0110226.

[14] C.S. Chu, P.M. Ho, and Y.C. Kao, Phys. Rev. D 60, 126003 (1999).

[15] A. Connes, Noncommutative Geometry (Academic, New York, 1996).

[16] A.H. Chamseddine, G. Felder, and J. Fröhlich, Commun. Math. Phys. 155, 205 (1993).

[17] P.M. Ho, Int. J. Mod. Phys. A 12, 923 (1997).

[18] A. Guth, Phys. Rev. D 23, 347 (1981).

[19] A.D. Linde, Phys. Lett. 129B, 177 (1983).

[20] C.S. Chu, J. Lukierski, and W.J. Zakrzewski, "Hermitian analyticity, IR/UV mixing and unitarity of noncommutative field theories," hep-th/0201144. 
[21] D.A. Eliezer and R.P. Woodard, Nucl. Phys. B325, 389 (1989); T.C. Cheng, P.M. Ho, and M.C. Yeh, ibid. B625, 151 (2002).

[22] N. Kaloper, M. Kleban, A. Lawrence, and S. Shenker, "Signatures of short distance physics in the cosmic microwave background," hep-th/0201158.

[23] N.D. Birrell and P.C.W. Davies, Quantum Fields in Curved Space (Cambridge University Press, Cambridge, England, 1982).

[24] F. Lizzi, G. Mangano, G. Miele, and M. Peloso, "Cosmological perturbations and short distance physics from Noncommutative Geometry," hep-th/0203099.

[25] G. Shiu and I. Wasserman, "On the Signature of Short Distance Scale in the Cosmic Microwave Background," hep-th/0203113.

[26] V.A. Rubakov, M.V. Sazhin, and A.V. Veryaskin, Phys. Lett.
115B, 189 (1982); L.F. Abbott and M.B. Wise, Nucl. Phys. B244, 541 (1984).

[27] V.F. Mukhanov, H.A. Feldman, and R.H. Brandenberger, Phys. Rep. 215, 203 (1992).

[28] A. Liddle and D. Lyth, Cosmological Inflation and LargeScale Structure (Cambridge University Press, Cambridge, England, 2000).

[29] R.H. Brandenberger and J. Martin, hep-th/0202142.

[30] V.F. Mukhanov, Pisma Zh. Éksp. Teor. Fiz. 41402 (1985) [JETP Lett. 41, 493 (1985)].

[31] V. Lukash, Sov. Phys. JETP 52, 807 (1980).

[32] D.H. Lyth, Phys. Rev. D 31, 1792 (1985).

[33] V.F. Mukhanov, Zh. Éksp. Teor. Fiz. 941 (1988) [Sov. Phys. JETP 67, 1297 (1988)]. 